\title{
High- and Low-Confinement Modes in Simple Magnetized Toroidal Plasmas
}

\author{
Paolo Ricci, ${ }^{1}$ B. N. Rogers, ${ }^{2}$ and S. Brunner ${ }^{1}$ \\ ${ }^{1}$ Centre de Recherches en Physique des Plasmas - École Polytechnique Fédérale de Lausanne, \\ Association EURATOM-Confédération Suisse, CH-1015 Lausanne, Switzerland \\ ${ }^{2}$ Department of Physics and Astronomy, Dartmouth College, Hanover, New Hampshire 03755, USA
}

(Received 10 November 2007; published 3 June 2008)

\begin{abstract}
Three-field simulations of interchange turbulence are presented for a simple magnetized toroidal plasma with a vertical magnetic field. The simulations show the presence of two turbulent regimes characterized by low $(L)$ and high $(H)$ confinement properties. We evaluate analytically the properties of the $L$ regime, obtaining expressions for the plasma gradients and for the density and heat fluxes that agree well with the simulations. By increasing the plasma source strength or reducing the vertical magnetic field, a transition to a $H$ regime occurs, in which a strong velocity shear limits the perpendicular transport with respect to the $L$ scaling and the plasma profiles steepen. The analytic estimate of the transition condition is in accord with the simulations.
\end{abstract}

DOI: 10.1103/PhysRevLett.100.225002

PACS numbers: 52.35.Ra, 52.30.Ex, 52.65.-y

We consider plasma turbulence in a simple magnetized torus (SMT) in which a vertical magnetic field $B_{v}$, superposed on a toroidal field $B_{\phi}$, creates helicoidal field lines with both ends terminating on the torus vessel. As in the scrape-off layer (SOL) of magnetic fusion devices, the turbulence driven by magnetic curvature and plasma gradients causes the plasma to diffuse in the radial direction, while at the same time it is progressively lost due to flows along the field lines. The SMT, employed since the 1980s in the context of tokamak preionization [1], is used for basic plasma physics studies [2,3]. The configuration facilitates the experimental study of low frequency instabilities and the related turbulence and cross-field transport, as it allows more detailed diagnostics and wider parameter scans than are usually possible in major confinement devices. Moreover, in addition to its intrinsic interest, the SMT's relative simplicity provides a useful test bed in which to explore the physics that presumably underlies transport in the SOL of more complex geometries.

In the present Letter, we describe three-field simulations of plasma turbulence in the SMT configuration. For concreteness we focus on the typical parameters of the toroidal plasma experiment (TORPEX) [3], confining an essentially electrostatic, $\beta \ll 1$, plasma with $T_{i} \ll T_{e}$. Furthermore, we restrict our attention to the cases in which the interchange-driven transport dominates over drift wave transport, i.e., $D_{D} / D_{I}<1$, where $D_{D}$ and $D_{I}$ are the anomalous diffusion coefficients due to drift waves and the interchange instability, respectively. According to mixing length estimates $D_{D} \sim c_{s} \rho_{s}^{2} / L_{p}$, where $L_{p}$ is the pressure scale length, $c_{s}=\sqrt{T_{e} / m_{i}}, \rho_{s}=c_{s} / \Omega_{i}$, and $\Omega_{i}=$ $e B_{0} /\left(m_{i} c\right)$. Later we show that $D_{I} \sim\left(c_{s} / k_{\Delta}\right) \sqrt{2 L_{p} / R}$, where $R$ is the major radius of the torus and $k_{\Delta}=2 \pi / \Delta$ the minimum perpendicular wavelength of the interchange instability, constrained by the return of the field lines in the poloidal plane, i.e., by the distance $\Delta=2 \pi R B_{v} / B_{\phi}=$ $L_{v} / N$ ( $N$ is the number of turns of a field line and $L_{v}$ the SMT height; assuming a high aspect ratio, we take $\Delta$ constant). Thus, interchange transport is expected to dominate over drift waves if $k_{\Delta} \rho_{s}^{2}\left[R /\left(2 L_{p}^{3}\right)\right]^{1 / 2}<1$.

From drift-reduced Braginskii equations [4], we derive simple two-dimensional fluid equations. We assume that $B_{v} \ll B_{\phi}$, so that $B \simeq B_{0} R / r$, and note that magnetic curvature is constant along a field line and equal to $r$. We denote the radial direction with $x$, the parallel direction with $z$, and the direction perpendicular to $x$ and $z$ with $y$. The Braginskii equations are integrated in the parallel direction to evolve the line-integrated density $n(x, y)=$ $\int \mathcal{N}(x, y, z) d z / L_{c}$, potential $\phi(x, y)=\int \Phi(x, y, z) d z / L_{c}$, and temperature $T_{e}(x, y)=\int \mathcal{T}_{e}(x, y, z) d z / L_{c}, \quad L_{c}=$ $2 \pi N R$ being the magnetic field line length. Reference [5] shows that the ion parallel flow at the sheath edge can be approximated as $\Gamma_{\|, i}=n c_{s} / 2$, and the electron flow as $\Gamma_{\|, e}=n c_{s} \exp \left(-e \phi / T_{e}+\Lambda\right) / 2$, with $\Lambda=$ $\log \sqrt{m_{i} /\left(2 \pi m_{e}\right)}$, where the presheath drops (mainly localized in a small region next to the sheath) in the evaluation of the $z$ integrals defining $n$ and $\phi$ are neglected. With the Boussinesq approximation for the polarization drift [6], the fluid equations are

$$
\begin{aligned}
\frac{\partial n}{\partial t}= & {\left[\frac{c \phi}{B_{0}}, n\right]+\frac{2 c}{e R B_{0}}\left(n \frac{\partial T_{e}}{\partial y}+T_{e} \frac{\partial n}{\partial y}-e n \frac{\partial \phi}{\partial y}\right) } \\
& +D \nabla^{2} n-\frac{\sigma n c_{s}}{R} \exp \left(\Lambda-e \phi / T_{e}\right)+S_{n}, \\
\frac{\partial \nabla^{2} \phi}{\partial t}= & {\left[\frac{c \phi}{B_{0}}, \nabla^{2} \phi\right]+\frac{2 B_{0}}{c m_{i} R}\left(\frac{T_{e}}{n} \frac{\partial n}{\partial y}+\frac{\partial T_{e}}{\partial y}\right)+\nu \nabla^{4} \phi } \\
& +\frac{\sigma c_{s} m_{i} \Omega_{i}^{2}}{e R}\left[1-\exp \left(\Lambda-e \phi / T_{e}\right)\right],
\end{aligned}
$$




$$
\begin{aligned}
\frac{\partial T_{e}}{\partial t}= & {\left[\frac{c \phi}{B_{0}}, T_{e}\right]+\frac{4 c}{3 e R B_{0}}\left(\frac{7}{2} T_{e} \frac{\partial T_{e}}{\partial y}+\frac{T_{e}^{2}}{n} \frac{\partial n}{\partial y}-e T_{e} \frac{\partial \phi}{\partial y}\right) } \\
& +k_{e} \nabla^{2} T_{e}-\frac{2}{3} \frac{\sigma T_{e} c_{s}}{R}\left[1.71 \exp \left(\Lambda-e \phi / T_{e}\right)-0.71\right] \\
& +S_{T},
\end{aligned}
$$

where $S_{n}$ and $S_{T}$ represent particle and heat sources, $\sigma=$ $R / L_{c}=\Delta /\left(2 \pi L_{v}\right)$, and $[a, b]=\partial_{x} a \partial_{y} b-\partial_{y} a \partial_{x} b$. A similar system of equations has been used in Ref. [7]. We denote equilibrium (i.e., time-averaged) quantities with the overbar and fluctuations with a tilde (e.g., $n=\bar{n}+\tilde{n}$ ).

Relative to the ideal growth rate, the linear growth $\gamma$ associated with Eqs. (1)-(3) is reduced at low $k_{y}$ because of sheath effects and at high $k_{y}$ because of diffusion. Herein, however, we focus on systems in which sheath effects do not affect strongly the linear properties of (1)(3), i.e., $k_{\Delta}^{2} \rho_{s}^{2} \gtrsim \sigma\left[L_{p} /(2 R)\right]^{1 / 2}$. Thus, the longest wavelength mode allowed in the system corresponds to the fastest growing instability, with a growth rate comparable to the interchange growth rate $\gamma \sim \sqrt{2} c_{s} / \sqrt{R L_{p}} \equiv \gamma_{0}$. The nonlinear equations (1)-(3) are solved with a numerical code developed from the ESEL code [8] that implements the algorithm described in Ref. [9]. We consider a rectangular domain with extension $L_{x}$ and $\Delta$ along $x$ and $y$, respectively. Dirichelet boundary conditions are imposed at $x=$ $0, L_{x}$, and, because of the flute character of the interchange mode, periodic boundary conditions are assumed along $y$. The simulations are started with small amplitude random noise in $n, \phi$, and $T_{e}$. The sources then introduce plasma and heat, increasing the plasma pressure and triggering the interchange instability. After a transient phase, a quasistationary state is reached in which the plasma, generated by the source and transported by the interchange dynamics in the radial direction, is eventually removed from the system by parallel losses. The results described here focus on this quasistationary state and its convergence has been checked by following simulations up to $t \gamma_{0} \simeq 4000$.

We normalize $n$ and $T_{e}$ to reference values $n_{0}$ and $T_{e 0}$, time $t$ to $R / c_{s 0}\left(c_{s 0}=\sqrt{T_{e 0} / m_{i}}\right)$, and lengths to $\rho_{s 0}=$ $c_{s 0} / \Omega_{i}$. We use $R=200, L_{v}=64, L_{x}=200, D=1$, $\nu=0.5, k_{e}=1$, and $\Lambda=3$. The sources are defined as $S_{n}=S_{T}=S_{0}\left\{S_{\mathrm{UH}} \exp \left[-\left(x-x_{\mathrm{UH}}\right)^{2} / \lambda_{\mathrm{UH}}^{2}\right]+S_{\mathrm{EC}} \exp [-(x-\right.$ $\left.\left.\left.x_{\mathrm{EC}}\right)^{2} / \lambda_{\mathrm{EC}}^{2}\right]\right\}\left(S_{\mathrm{UH}}=1.5, S_{\mathrm{EC}}=1, \lambda_{\mathrm{UH}}=5, \lambda_{\mathrm{EC}}=2.5\right.$, $\left.x_{\mathrm{UH}}=35, x_{\mathrm{EC}}=15\right)$, in order to mimic the upper hybrid (UH) and electron cyclotron (EC) sources in TORPEX [10]. The simulation results are insensitive to the details of the plasma source. We perform a set of simulations in which we vary $\sigma$ and $S_{0}(\sigma=0.03,0.04,0.05,0.07,0.08$, $\left.S_{0}=0.01,0.025,0.05,0.1,0.25,0.5,1,2,4,8\right)$.

The simulations show the presence of two turbulent regimes. Typical snapshots of $\phi$ from a simulation in the first regime, denoted as the low-confinement mode ( $L$ mode), are shown in the upper panels in Fig. 1. In this regime, background shear flow effects are negligible, and the interchange instability displays an intermittent character, with the emission of plasma blobs that transfer plasma from the source region to the low-field region while the plasma is simultaneously drained by parallel absorption. The profiles of $\bar{n}, \bar{T}_{e}$, and $\bar{\phi}$ show an exponential decay on the low-field side where the interchange dynamics is active (i.e., for $x>x_{S}, x_{S}$ denoting the right-most peak in density and temperature). By increasing $S_{0}$ or reducing $\sigma$, a new turbulent regime is reached, denoted as the $H$ mode, in which a strong $\mathbf{E} \times \mathbf{B}$ shear flow appears. This flow limits the perpendicular diffusion coefficient, thereby causing a reduction of the flux with respect to the $L$-mode estimate and steeper plasma profiles. The lower panels in Fig. 1 show typical snapshots and profiles in the $H$-mode regime.

We first consider turbulence in the $L$-mode regime. Let us estimate the $y$-averaged cross-field particle transport $\Gamma_{n}=R\left\langle\overline{\tilde{n} \partial_{y} \tilde{\phi}}\right\rangle_{y}$. We first note that in the quasistationary state, since $L_{n}<R$ and $\sigma<1$, the dominant terms in Eq. (1) lead to $\partial_{t} n+R[\phi, n] \simeq 0$. Since in the $L$ mode the shear flow plays a negligible role, one has $\partial_{y} \tilde{\phi} \sim$ $\gamma_{0} \tilde{n} /\left(R \partial_{x} \bar{n}\right)$, which is well satisfied by simulation data. Turbulence saturation occurs when the instability drive is removed from the system, i.e., when $\partial_{x} \bar{n} \sim \partial_{x} \tilde{n}$. By following standard local theory methods (outlined, e.g., in Ref. [11] for drift waves), it is possible to estimate the typical radial extension of the interchange mode in the main regime of interest here: $k_{y} L_{p}>1$. The leading order (i.e., ideal MHD interchange) terms in the system (1)-(3) reduce to $\partial_{x}^{2} \tilde{\phi}-k_{y}^{2}[1+G(x)] \tilde{\phi}=0$, where $G(x)=$ $-2 R \partial_{x} p_{e} /\left(n \gamma^{2}\right) . \quad$ By Taylor-expanding $\quad G(x) \simeq G_{0}+$ $G_{0}^{\prime \prime}\left(x-x_{0}\right)^{2} / 2$, with $x_{0}$ the point of instantaneous steepest pressure gradient, $G_{0}=G\left(x_{0}\right)$, and $G_{0}^{\prime \prime}=\left.\partial_{x}^{2} G(x)\right|_{x=x_{0}} \sim$ $2 R T_{e} /\left(\gamma^{2} L_{p}^{3}\right)$, one is lead to a harmonic oscillator equation for $\tilde{\phi}$. In the limit $k_{y} L_{p}>1$, one has $\gamma=\left.\gamma_{0}\right|_{x=x_{0}}$ and $\tilde{\phi} \propto$ $\exp \left[-a\left(x-x_{0}\right)^{2} / 2\right]$, with $a=k_{y}\left(\left|G_{0}^{\prime \prime}\right| / 2\right)^{1 / 2} \sim k_{y} / L_{p}$. The typical radial extension of the interchange mode is
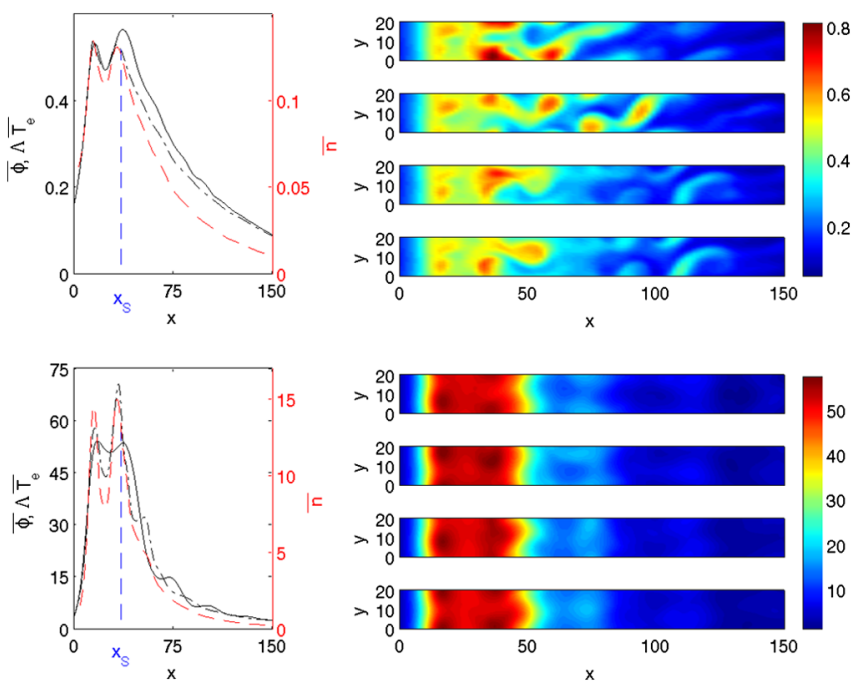

FIG. 1 (color online). $\quad \bar{\phi}$ (solid black curve), $\bar{T}_{e}$ (dashed-dotted black curve), and $\bar{n}$ (dashed red curve) and typical $\phi$ snapshots for $S_{0}=0.01$ (upper panels) and $S_{0}=8$ (lower panels). $\sigma=0.05$. 
thus given by $1 / \sqrt{a} \sim\left(L_{p} / k_{y}\right)^{1 / 2}$, and, by considering the dominant mode $k_{y}=k_{\Delta}$, one estimates $\partial_{x} \tilde{n} \sim \tilde{n}\left(k_{\Delta} / L_{p}\right)^{1 / 2}$, which finally gives $\tilde{n} \sim \bar{n}\left(L_{p} / k_{\Delta}\right)^{1 / 2} / L_{n}$ (comparison with simulation data shows that the latter overestimates the density fluctuations by a factor of $\simeq 2$ ). The evaluation of $\tilde{n}$ and $\partial_{y} \tilde{\phi}$ leads to estimates of the radial density flux in the $L$-mode regime as $\Gamma_{n, L}=\bar{n}\left(2 R L_{p} \bar{T}_{e}\right)^{1 / 2} /\left(L_{n} k_{\Delta}\right)$ and to an expression for the temperature flux $\Gamma_{T, L}=$ $\left(2 R L_{p} \bar{T}_{e}^{3}\right)^{1 / 2} /\left(L_{T} k_{\Delta}\right)$ [or equivalently, a diffusion coefficient $\left.D_{I}=\left(2 R L_{p} \bar{T}_{e}\right)^{1 / 2} / k_{\Delta}\right]$. We note that the KelvinHelmholtz $(\mathrm{KH})$ instability could provide an alternative saturation mechanism, but numerical tests (discussed later) show that $\mathrm{KH}$ instability does not play a significant role in the $L$ mode. In Fig. 2, we compare the values of $\Gamma_{n}$ and $\Gamma_{T}$ from the simulations with the $L$-mode estimates, both quantities being evaluated at $x=x_{S}$, with $L_{n}\left(L_{T}\right)$ deduced from the radial distance between $n_{\max }$ and $n_{\max } / 2\left(T_{e, \max }\right.$ and $T_{e, \max } / 2$ ). Two sets of simulations are shown: In the first set, corresponding to all values of $S_{0}$ for the highest $\sigma$ and small values of $S_{0}$ for small $\sigma$, both $\Gamma_{n}$ and $\Gamma_{T}$ follow the predicted scaling laws. These simulations belong to the $L$-mode regime. In the second set, i.e., small $\sigma$ and high $S_{0}$, the transport level is smaller than the predictions. Figure 3(a) shows that the discrepancies with the $L$-mode predictions are related to shear flow stabilization. The $H$ factor, defined as $H_{n}=\Gamma_{n, L} / \Gamma_{n}$ and $H_{T}=\Gamma_{T, L} / \Gamma_{T}$, is plotted as a function of $\gamma_{0} / v_{\mathbf{E} \times \mathbf{B}}^{\prime}$, where $\gamma_{0}$ is evaluated at $x=x_{S}$, while $v_{\mathbf{E} \times \mathbf{B}}^{\prime}=\partial_{x}^{2} \phi$ denotes the maximum shear flow on the low-field side $\left(H_{n}\right.$ and $H_{T}$ vary by $\sim 15 \%$ along $x$ ). For $\gamma_{0} / v_{\mathbf{E} \times \mathbf{B}}^{\prime}>0.7$ the simulations fall in the $L$-mode regime, where the previously obtained scaling is obeyed: $\Gamma_{n} \simeq \alpha \Gamma_{n, L}$ and $\Gamma_{T} \simeq \alpha \Gamma_{T, L}$, with $\alpha=0.3 \pm 0.03$. The confinement improves when the shear flow increases: We define the $L-H$ transition to occur when $\Gamma_{n} \simeq \alpha \Gamma_{n, L} / 3$ and $\Gamma_{T} \simeq \alpha \Gamma_{T, L} / 3$; this takes place for $\gamma_{0} / v_{\mathbf{E} \times \mathbf{B}}^{\prime} \simeq 0.5$ (vertical purple lines in Fig. 3).

We now show that, in the $L$-mode regime, $L_{n}$ and $L_{T}$ depend only on the geometric parameters of the SMT. As illustrated in Fig. 1, the potential is constrained to follow $\phi \simeq \Lambda T_{e}$ due to the exponential dependence of $\Lambda-\phi / T_{e}$ in Eqs. (1)-(3). By integrating Eqs. (1) and (2) along $y$, time averaging, and neglecting diffusivity, one obtains
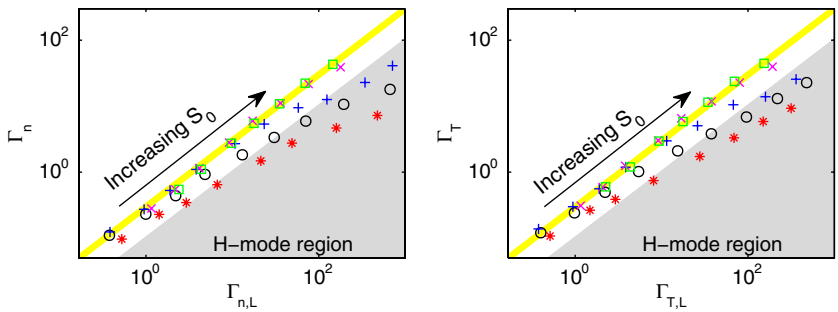

FIG. 2 (color online). $\Gamma_{n}$ and $\Gamma_{T}$ vs $\Gamma_{n, L}$ and $\Gamma_{T, L}$, for $\sigma=$ 0.03 (red stars), $\sigma=0.04$ (black circles), $\sigma=0.05$ (blue crosses), $\sigma=0.07$ (magenta $\times$ ), and $\sigma=0.08$ (green squares). The shaded area is the $H$-mode region.

$$
\frac{\partial \Gamma_{n}}{\partial x} \simeq S_{n}-\sigma \bar{n} \bar{T}_{e}^{1 / 2}, \quad \frac{\partial \Gamma_{T}}{\partial x} \simeq S_{T}-\frac{2}{3} \sigma \bar{T}_{e}^{3 / 2} .
$$

Within the $L$ regime, Eqs. (4) admit solutions of the form $\bar{n}=n_{\max } \exp \left[\left(x-x_{S}\right) / L_{n, L}\right]$ and $\bar{T}_{e}=$ $T_{e, \max } \exp \left[\left(x-x_{S}\right) / L_{T, L}\right]$ on the low-field (right) side of the source free region, i.e., for $x>x_{S}$ (where $x_{S} \simeq 36$ in the present simulations). By substituting the expression of $\Gamma_{n, L}$ and $\Gamma_{T, L}$ for $\Gamma_{n}$ and $\Gamma_{T}$ in Eqs. (4), we obtain $\alpha \sqrt{2 L_{p, L}} / L_{n, L}\left[1 / L_{n, L}+1 /\left(2 L_{T, L}\right)\right]=1 /\left(L_{v} \sqrt{R}\right) \quad$ and $\alpha \sqrt{2 L_{p, L}} / L_{T, L}^{2}=4 /\left(9 L_{v} \sqrt{R}\right)$, thus finding $L_{n, L} \simeq$ $0.58\left(L_{v} \sqrt{R}\right)^{2 / 3}$ and $L_{T, L} \simeq 0.74\left(L_{v} \sqrt{R}\right)^{2 / 3}$, which, as claimed, depend only on the geometry of the machine. In Fig. 3(b), we plot $L_{T, L} / L_{T}$ vs $\gamma_{0} / v_{\mathbf{E} \times \mathbf{B}}^{\prime}$ : As expected, in the case of negligible shear flow, $L_{n}$ and $L_{T}$ do not depend on $S_{0}, \sigma$, and $x$ (within $15 \%$ oscillation); in the $H$ mode, the reduction of the diffusion coefficient with respect to $D_{I}$ leads to smaller $L_{n}$ and $L_{T}$.

We now turn to the $L-H$ transition threshold. Insight into the nature of the velocity shear is given by the time-averaged and $y$-integrated Eq. (2), $R \partial_{x}^{2}\left\langle\tau_{x y}\right\rangle_{y}=$ $\sigma\left\langle\overline{\sqrt{T_{e}}\left[1-e^{\left(\Lambda-\phi / T_{e}\right)}\right]}\right\rangle_{y}$, where $\tau_{x y}=\overline{\partial_{x} \tilde{\phi} \partial_{y} \tilde{\phi}}$ is the $x y$ component of the turbulence-induced Reynold stress (RS), having neglected $\nu \nabla^{4} \phi$ and approximated $\left\langle\overline{T_{e} \partial_{y} \log n}\right\rangle_{y} \simeq$ 0 . Since deviations of the potential from $\phi=\Lambda T_{e}$ are due to the presence of the RS, shear flow can be related either to a sheared temperature profile (STP), i.e., $\partial_{x}^{2} \phi \simeq \Lambda \partial_{x}^{2} T_{e}$, or to RS-induced shear. In both cases, the amplitude of the shear flow is limited by the KH instability. Reference [12] evaluates the KH growth rate in the case of a Bickley jet and shows that $\mathrm{KH}$ is stable if $k_{y} L_{\phi}>\zeta(\zeta \simeq 2)$. Analogously, in our system, the $\mathrm{KH}$ instability imposes $k_{y} L_{\phi}>\delta$, where the most stringent criterion for stability is obtained for $k_{y}=k_{\Delta}$, i.e., $L_{\phi}>\delta \Delta /(2 \pi)$. In most of our simulations, shear flow tends to reach the maximum value allowed by the $\mathrm{KH}$ instability, i.e., $L_{\phi} \simeq \delta \Delta /(2 \pi)$, so that, in general, $v_{\mathbf{E} \times \mathbf{B}}^{\prime} \simeq \chi \phi_{\max } / \Delta^{2}$, with $\chi \simeq 2.5(\delta=2 \pi / \sqrt{\chi})$.

In the $L$ regime, one observes that $\partial_{x}^{2} \bar{\phi}>\Lambda \partial_{x}^{2} \bar{T}_{e}$ and thus that shear flow is mostly related to the RS term. However, $L$-mode simulations are not significantly affected when the $y$-averaged RS term is artificially removed, thus showing that shear flow plays no significant role in this regime. When approaching the $H$ mode, the
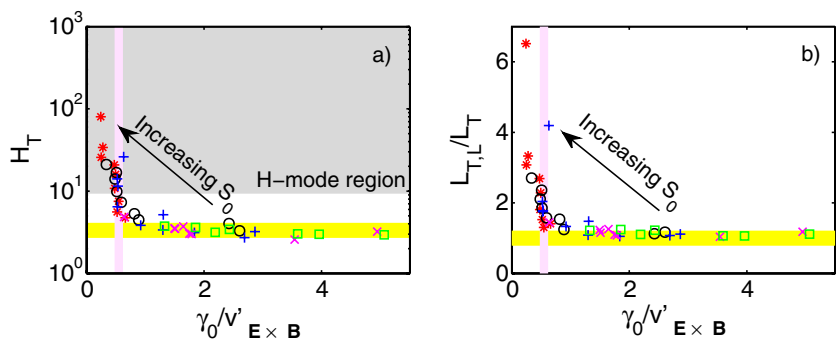

FIG. 3 (color online). $H_{T}$ and $L_{T, L} / L_{T}$ vs $\gamma_{0} / v_{\mathbf{E} \times \mathbf{B}}^{\prime}$; same notation as Fig. 2. The plots of $H_{n}$ and $L_{n, L} / L_{n}$ (not shown) reveal essentially identical dependences. 


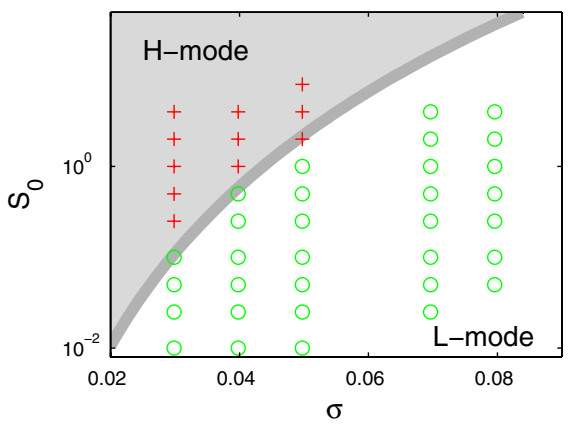

FIG. 4 (color online). $L-H$ threshold from Eq. (5) and simulations falling in the $H$-mode (red crosses) and $L$-mode (green circles) regimes.

shear flow amplitude increases, and the relative importance of the contribution due to the STP is enhanced. At the $L-H$ threshold, one then observes a comparable contribution to the shear flow from the RS and the STP term, and thus $\partial_{x}^{2} \phi \sim \Lambda \partial_{x}^{2} T_{e}$, i.e., $L_{T} \sim L_{\phi}$.

Assuming that $L_{n} \sim L_{T}$ and $\phi_{\max } \sim \Lambda T_{e, \max }$ leads to $\gamma_{0} / v_{\mathbf{E} \times \mathbf{B}}^{\prime} \simeq 2 \Delta^{3 / 2} /\left(\chi \Lambda \sqrt{R T_{e, \max }}\right)$ at the $L-H$ threshold. The value $T_{e, \max }$ can be deduced by integrating Eq. (4) along the $x$ direction across the entire domain. An exponential dependence of the temperature is assumed for $x>$ $x_{S}$ and, to take into account the diffusion-dominated dynamics for $x<x_{S}$, a shape factor $\xi$ is introduced such that $\int_{0}^{x_{S}} T_{e}^{3 / 2} d x=\xi T_{e, \max }^{3 / 2} x_{S} \quad(\xi \simeq 0.5)$. One concludes that $T_{e, \max }^{3 / 2} \simeq \int S_{T} d x /\left[\sigma\left(2 \xi x_{S} / 3+4 L_{T} / 9\right)\right]$. The $L-H$ transition occurs for $\gamma_{0} / v_{\mathbf{E} \times \mathbf{B}}^{\prime} \simeq 0.5$, which leads to

$$
\frac{2 \Delta^{11 / 6}}{\chi \Lambda \sqrt{R}}\left(\frac{4 / 9 \Delta+2 \xi x_{S} / 3}{2 \pi L_{v} \int S_{T} d x}\right)^{1 / 3} \simeq 0.5 \text {. }
$$

Equation (5) can be used to find the relation between $S_{0}$ and $\Delta$ at the $L-H$ threshold. With the simulation parameters, the threshold condition can be written as $S_{0}=8.54 \times$ $10^{-8} \Delta^{11 / 2}(1+0.039 \Delta)$. In Fig. 4 , the $L-H$ threshold prediction shows good agreement with the simulations.

Deep into the $H$ mode, the relative contribution to the shear flow from the RS and the STP remain comparable. In this regime the RS is essential. Contrary to the $L$-mode simulations, if the $y$-averaged RS term is artificially removed from the model, the $H$-mode simulations are deeply affected. One observes the development of radial streamers that continuously transport plasma from the source region to the right boundary of the domain, degrading the perpendicular confinement. This is related to the fact that the RS is essential for the formation of sheared flows and the breakup of radial streamers via, for example, the $\mathrm{KH}$ instability. We also point out that, in the $H$ mode, the regions within the barrier in which the temperature profile flattens, $\partial_{x} \bar{T}_{e} \simeq 0$, are centered on the location where velocity shear is minimum, $\partial_{x}^{2} \bar{\phi} \simeq 0$, i.e., where the interchange mode is not stabilized.

We finally note that the energy confinement time can be estimated as $\tau\left(L_{T}\right)=\int T_{e} d x / \int S_{T} d x \simeq\left(L_{T}+\xi x_{S}\right) /$ $\left[\sigma\left(\int S_{T} d x\right)^{1 / 3}\left(2 \xi x_{S} / 3+4 L_{T} / 9\right)^{2 / 3}\right]$, with the $L$-scaling prediction being $\tau_{L}=\tau\left(L_{T, L}\right)$. If one attempts to evaluate the gain in the confinement time, one observes that $\tau / \tau_{L} \leq$ 1 for $L_{T} / L_{T, L} \leq 1$. Contrary to the tokamak case, the $H$-mode confinement time thus decreases with respect to the $L$ scaling. This results from the fact that in a SMT all field lines are subject to higher parallel sheath losses as $T_{e}$ increases in the $H$ mode.

The intermittent turbulence recently reported in TORPEX [13] corresponds, according to the theory outlined here, to the $L$-mode regime. The estimate of $D_{D} / D_{I}$ shows that the drift wave instability becomes more important for small $B_{v}$ or steep plasma profiles, which are the conditions typical for the $H$ regime to appear. Fully threedimensional simulations are required to study the effect of the drift wave dynamics on the system and how it is impacted by the sheared flows discussed here. Strong similarities exist between the tokamak and the SMT $L-H$ transition. In fact, as in the tokamak, the $H$ mode is driven by the increase of the input power and is characterized by strong shear flows leading to a steeper density and temperature gradients, higher peak density and temperature, and reduced radial diffusion coefficients. However, contrary to the tokamak case, the confinement time is reduced in the SMT, as a result of increased sheath losses.

We gratefully acknowledge O.E. Garcia for providing the original version of the fluid code used here as well as many useful discussions with A. Diallo, A. Fasoli, I. Furno, B. Labit, M. Podestà, F. M. Poli, and C. Theiler. P. R. is supported by a EURATOM fusion fellowship and B. R. by the U.S. DOE and NSF.

[1] S. Nakao et al., Phys. Lett. 96A, 405 (1983).

[2] E. D. Zimmerman and S. C. Luckhardt, J. Fusion Energy 12, 289 (1993); K. Rypdal and S. Ratynskaia, Phys. Rev. Lett. 94, 225002 (2005); P. K. Sharma and D. Bora, Plasma Phys. Controlled Fusion 37, 1003 (1995); J.C. Perez et al., Phys. Plasmas 13, 032101 (2006); C. Riccardi et al., Plasma Phys. Controlled Fusion 36, 1791 (1994).

[3] A. Fasoli et al., Phys. Plasmas 13, 055902 (2006).

[4] A. Zeiler et al., Phys. Plasmas 4, 2134 (1997).

[5] P. C. Stangeby, The Plasma Boundary of Magnetic Fusion Devices (Institute of Physics, Berkshire, 2000).

[6] C. Q. Yu et al., Phys. Plasmas 13, 042508 (2006).

[7] N. Bisai et al., Phys. Plasmas 11, 4018 (2004).

[8] V. Naulin et al., Phys. Rev. Lett. 81, 4148 (1998); O.E. Garcia et al., Phys. Rev. Lett. 92, 165003 (2004).

[9] V. Naulin and A. H. Nielsen, SIAM J. Sci. Comput. 25, 104 (2003).

[10] M. Podestà et al., Plasma Phys. Controlled Fusion 48, 1053 (2006).

[11] B. N. Rogers and W. Dorland, Phys. Plasmas 12, 062511 (2005).

[12] D. Biskamp, Magnetic Reconnection in Plasma (Cambridge University Press, Cambridge, England, 2000).

[13] I. Furno et al., Phys. Rev. Lett. 100, 055004 (2008). 